UCD 517.9

\title{
GLOBAL ROBUST EXPONENTIAL STABILITY FOR HOPFIELD NEURAL NETWORKS WITH NON-LIPSCHITZ ACTIVATION FUNCTIONS*
}

\section{ГЛОБАЛЬНА РОБАСТНА ЕКСПОНЕНЦАЛЬНА СТІЙКІСТЬ ДЛЯ НЕЙРОННИХ МЕРЕЖ ХОПФІЛЬДА З НЕЛІПШИЦЕВОЮ ФУНКЦІЕЮ АКТИВАЦЇ̈}

\author{
Hongtao Yu \\ College Inform. Sci. and Engineering, Yanshan Univ. \\ Qinhuangdao 066004, China \\ e-mail:yu5771@163.com
}

\section{Huaiqin Wu}

College Sci., Yanshan Univ.

Qinhuangdao 066001, China

e-mail: huaiqinwu@ysu.edu.cn

This paper is concerned with the problem of the global robust exponential stability for Hopfield neural networks with norm-bounded parameter uncertainties and inverse Hölder neuron activation functions. By applying Brouwer degree properties and some analysis techniques, the existence and uniqueness of the equilibrium point are investigated. Based on the Lyapunov stability theory, a global robust exponential stability criterion is derived in terms of linear matrix inequality (LMI). Two numerical examples are provided to demonstrate the effectiveness and validity of the proposed robust stability results.

Розглянуто задачу глобальної робастної експоненціальної стійкості для нейронних мереж Хопфільда з обмеженими за нормою параметричною невизначеністю та оберненими функціями Гельдера нейронної активації. Використовуючи властивості ступеня Брауера та результати з аналізу, вивчено питання існування та единості точки рівноваги. Критерій глобальної робастної експоненціальної стійкості в термінах лінійної матричної нерівності отримано з використанням теорії стійкості Ляпунова. Наведено два числових приклади для ілюстрациї ефективності та дієвості наведених результатів.

1. Introduction. In recent years, there has been increasing interest in the dynamic analysis of artificial neural networks. Among the most popular models in the previous literatures are the Hopfield neural networks (HNNs) proposed by Hopfield. This network has attracted numerous attention due to their promising application in the various engineering problems, such as, classification of patterns, solving optimization problems, designing associative memory. It has been observed that such applications greatly rely on the dynamical analysis of the neural network, in particular, the stability analysis of the neural network. As is well known, when designing neural network, it is central to investigate stability problem of neural networks. However, in the process of implementations of neural networks, parametric uncertainty which often breaks the

${ }^{*}$ This work was supported by the Natural Science Foundation of Hebei Province of China (A2011203103) and the Hebei Province Education Foundation of China (2009157).

(C) Hongtao Yu, Huaiqin $\mathrm{Wu}, 2012$

ISSN 1562-3076. Нелінійні коливання, 2012, m. 15, N으 1 
stability of a neural network can be commonly encountered due to the modeling inaccuracies and changes in the environment of the model. For example, in the practical application of neural networks, some vital data such as the neuron firing rates and the weight coefficients are usually acquired and processed by means of the statistical estimates. Thus, the robust stability analysis of different uncertain neural networks in the presence of parametric uncertainties has gained much research attention, see, e.g. [1-21] and the references therein. In a general way, there are two forms of parametric uncertainties, namely the interval uncertainty and the norm-bounded uncertainty. Based on matrix norms and Lyapunov stability theory, Refs. [1-6] derived some delay-independent or delay-dependent conditions of the existence, uniqueness and robust stability for interval uncertain HNNs with constant time delay or interval time-varying delay in terms of LMI. By applying matrix decomposition method, Halanay inequality and LMI techniques, Refs. [7, 8] established some delay-independent criteria for the robust stability for uncertain HNNs with multiple time-varying delays and continuously distributed delays. By using Jensen's integral inequality and Lyapunov-Krasovskii method, Refs. [9-11] achieved delay-dependent criteria of the robust stability for norm-bounded uncertain HNNs with time-varying delay. By applying the free weight method, LMI and Jensen's integral inequality techniques, Refs. [1217] proposed some delay-dependent criteria of the robust stability for norm-bounded uncertain HNNs with multiple time-varying delay. In addition, by using Jensen's integral inequality and LMIs, Refs. [18-20] proposed some delay-dependent robust stability criteria for HNNs with time-varying delay and linear fractional uncertainties and nonlinear uncertainties. Based on Brouwer degree properties, Refs [21] proved the existence of the equilibrium point of the interval neural network model with delays and inverse Hölder neuron activation functions, and by applying Lyapunov functional approach presented a sufficient condition which is used to ensure the interval robust stability of the network in terms of LMIs.

It should be noted, in the existing literature, almost all results on the robust stability of neural networks with parametric uncertainties are conducted under some special assumptions on neuron activation functions. These assumptions frequently include those such as Lipschitz, bounded and/or monotonic increasing property. To the best of our knowledge, there are few papers to deal with the global robust stability for neural networks with non-Lipschitz activation functions. However, many neural networks without Lipschitz continuous neuron activation functions frequently appear in the theoretical study of dynamics of neural networks. In addition, in order to solve a lot of practical engineering problems, whether neural networks with non-Lipschitz activation functions is stable or robust stable should be determined too. Hence giving the conditions of the robust stability for neural networks without Lipschitz continuous activation functions is very valuable in both theory and practice.

Motivated by the preceding discussion, the aim of this paper is to study the global robust exponential stability of HNNs with norm-bounded parameter uncertainties and inverse Hölder neuron activation functions. a sufficient condition will be derived to ensure the network to be globally robustlly exponential stable for all admissible parameter uncertainties. The rest of this paper is organized as follows. In Section 2, the model formulation and some preliminaries is given. The main result are stated in Section 3. In Section 4, two numerical examples are presented to demonstrate the effectiveness and validity of the proposed stability results. Finally, some conclusions are made in Section 5.

Notations. The notations used throughout this paper are standard. $A^{T}$ and $A^{-1}$ denote the transpose and the inverse of any square matrix $A . A>0(A<0)$ means that $A$ is positive definite negative definite. $R$ denotes the set of real numbers, $R^{n}$ denotes the $\mathrm{n}$-dimensional 
Euclidean space, $R^{m \times n}$ denotes the set of all $m \times n$ real matrices. $\mathcal{I}$ denotes the identity matrix with appropriate dimension. Given the column vectors $x=\left(x_{1}, \ldots, x_{n}\right)^{T} \in R^{n}$, the norm is the Euclidean vector norm, i.e., $\|x\|=\left(\sum_{i=1}^{n} x_{i}^{2}\right)^{\frac{1}{2}}$.

2. Model of neural network and preliminaries. Consider the following HNNs with parametric uncertainties described by the differential equation system

$$
\frac{d x}{d t}=-(D+\Delta D(t)) x(t)+(A+\Delta A(t)) g(x(t))+I,
$$

where $x(t)=\left(x_{1}(t), \ldots, x_{n}(t)\right)^{T}$ is the vector of neuron states at time $t ; D=\operatorname{diag}\left(d_{1}, \ldots, d_{n}\right)$ is an $n \times n$ constant diagonal matrices, $d_{i}>0, i=1, \ldots, n$, are the neural self-inhibitions; $A=$ $=\left(a_{i j}\right)_{n \times n}$ is an $n \times n$ interconnection matrix; $g(x)=\left(g_{1}\left(x_{1}\right), \ldots, g_{n}\left(x_{n}\right)\right)^{T}, g_{i}, i=1, \ldots, n$, are called the neuron activation functions; $I=\left(I_{1}, \ldots, I_{n}\right)^{T}$ denots the external input. $\Delta D(t)=$ $=\operatorname{diag}\left(\triangle d_{1}(t), \ldots, \triangle d_{n}(t)\right)$ and $\Delta A(t)=\left(\triangle a_{i j}(t)\right)_{n \times n}$ are continuous matrix-valued functions of $t$, and are used to denote the parametric uncertainties in the network.

Correspondingly, HNNs without parametric uncertainties

$$
\frac{d x}{d t}=-D x(t)+A g(x(t))+I
$$

is called as the reference neural network of (1).

The parametric uncertainties $\Delta D(t)$ and $\Delta A(t)$ are assumed to satisfy:

$A_{1}: \triangle d_{i}(t): R \rightarrow R$ is a continuous function, and satisfies $\left|\triangle d_{i}(t)\right|<d_{i}, i=1,2, \ldots, n$.

$A_{2}: \Delta A(t)=H F(t) E$, where $H$ and $E$ are known constant matrices with appropriate dimensions. The uncertain matrix $F(t)$ is an unknown time varying matrix with Lebegue measurable elements, and satisfies

$$
F^{T}(t) F(t) \leq \mathcal{I} \quad \forall t \in R
$$

Definition 2.1. The equilibrium point $x^{*}$ of the system (2) is said to be globally exponentially stable with convergence rate $\gamma$, if there are positive constants $\gamma, T$ and $\beta$, such that for any solution $x\left(t, 0, x_{0}\right)$ of the system (2) with initial value $x(0)=x_{0}$,

$$
\left\|x\left(t, 0, x_{0}\right)-x^{*}\right\| \leq \beta e^{-\gamma t}, \quad t \geq T .
$$

If the equilibrium point $x^{*}$ of the system (1) is globally exponentially stable, then the system (2) is said to be globally robustly exponentially stable.

Definition 2.2. A continuous function $\mathcal{G}: R \rightarrow R$, is said to be an $\alpha$-inverse Hölder function, if

(i) $\mathcal{G}$ is a monotonic nondecreasing function,

(ii) for any $\rho \in R$, there exist constants $q_{\rho}>0$ and $r_{\rho}>0$ which are correlated with $\rho$, satisfying

$$
|\mathcal{G}(\theta)-\mathcal{G}(\rho)| \geq q_{\rho}|\theta-\rho|^{\alpha} \quad \forall|\theta-\rho| \leq r_{\rho},
$$

where $\alpha>0$ is a constant. 
The class of $\alpha$-inverse Hölder functions is denoted by $\mathcal{I} \mathcal{L}(\alpha)$. When $\alpha=1,1$-inverse Hölder functions are called to be inverse Lipschitz functions. It is easy to check $\mathcal{G}(\theta)=\arctan \theta \in$ $\in \mathcal{I} \mathcal{L}(1), \mathcal{G}(\theta)=\theta^{3} \in \mathcal{I} \mathcal{L}(3)$.

Remark 1. It is obvious that $\alpha$-inverse Hölder functions are a class of non-Lipschitz functions.

Lemma 2.1 [22]. If $\mathcal{G}(\theta) \in \mathcal{I} \mathcal{L}(\alpha)$, then for any $\rho_{0} \in R$, we have

$$
\int_{\rho_{0}}^{+\infty}\left[\mathcal{G}(\theta)-\mathcal{G}\left(\rho_{0}\right)\right] d \theta=\int_{\rho_{0}}^{-\infty}\left[\mathcal{G}(\theta)-\mathcal{G}\left(\rho_{0}\right)\right] d \theta=+\infty
$$

Lemma 2.2 [23]. If $\mathcal{G}(\theta) \in \mathcal{I} \mathcal{L}(\alpha)$ and $\mathcal{G}(0)=0$, then there exist constants $q_{0}>0$ and $r_{0}>0$, such that

$$
|\mathcal{G}(\theta)| \geq q_{0}|\theta|^{\alpha} \quad \forall|\theta| \leq r_{0}
$$

Moreover,

$$
|\mathcal{G}(\theta)| \geq q_{0} r_{0}^{\alpha} \quad \forall|\theta| \geq r_{0}
$$

Let $\Omega$ be a nonempty, bounded and open subset of $R^{n}$. The closure of $\Omega$ is denoted by $\bar{\Omega}$, and the boundary of $\Omega$ is denoted by $\partial \Omega$.

Lemma 2.3 [24]. (1). Let $\mathcal{H}:[0,1] \times \bar{\Omega} \rightarrow R^{n}$ be a continuous mapping. If $p \bar{\in} \mathcal{H}(\lambda, \partial \Omega)$ for all $\lambda \in[0,1]$, then Brouwer degree $\operatorname{deg}(\mathcal{H}(\lambda, \cdot), \Omega, p)$ is constant $(\forall \lambda \in[0,1])$. In this case, $\operatorname{deg}(\mathcal{H}(0, \cdot), \Omega, p)=\operatorname{deg}(\mathcal{H}(1, \cdot), \Omega, p)$.

(2) Let $\mathcal{H}: \bar{\Omega} \rightarrow R^{n}$ be a continuous mapping. If $\operatorname{deg}(\mathcal{H}, \Omega, p) \neq 0$, then the equation $\mathcal{H}(x)=p$ has at least a solution in $\Omega$.

Lemma 2.4 (Schur complement). Given constant matrices $\Sigma_{1}, \Sigma_{2}$ and $\Sigma_{3}$ with appropriate dimensions, where $\Sigma_{1}=\Sigma_{1}^{T}$ and $\Sigma_{2}=\Sigma_{2}^{T}>0$, then

$$
\Sigma_{1}+\Sigma_{3}^{T} \Sigma_{2}^{-1} \Sigma_{3}<0 \Leftrightarrow\left[\begin{array}{cc}
\Sigma_{1} & \Sigma_{3}^{T} \\
\Sigma_{3} & -\Sigma_{2}
\end{array}\right]<0, \quad \text { or } \quad\left[\begin{array}{cc}
-\Sigma_{2} & \Sigma_{3} \\
\Sigma_{3}^{T} & \Sigma_{1}
\end{array}\right]<0
$$

Lemma 2.5. For matrices $P \in R^{n \times n}, M \in R^{n \times k}, N \in R^{l \times n}$ and $F \in R^{k \times l}$ with $P>0$, $F^{T} F \leq \mathcal{I}$, and scalar $\varepsilon>0$, the following matrix inequality holds:

$$
P M F N+(M F N)^{T} P \leq \varepsilon P M M^{T} P+\varepsilon^{-1} N^{T} N .
$$

\section{Main results.}

Theorem 3.1. Suppose $g_{i} \in \mathcal{I} \mathcal{L}(\alpha), i=1,2, \ldots, n$. Under the assumptions $A_{1}$ and $A_{2}$, if there exists a positive diagonal matrix $P=\operatorname{diag}\left(p_{1}, \ldots, p_{n}\right)$ and a scalar $\varepsilon>0$ such that

$$
\mathbb{E}=\left[\begin{array}{cc}
P A+A^{T} P+\varepsilon^{-1} E^{T} E & P H \\
H^{T} P & -\varepsilon^{-1} \mathcal{I}
\end{array}\right]<0
$$


then the neural network (1) has a unique equilibrium point which is globally exponentially stable, i.e., the reference neural network (2) is globally robustly exponentially stable.

Proof. The proof is devided into three steps.

Step 1: In this step, the proof of existence for the equilibrium point will be given.

Let $\mathcal{H}(x, t)=(D+\Delta D(t)) x-(A+\Delta A(t)) g(x)-I . x^{*} \in R^{n}$ is an equilibrium point of the system (1) if and only if $\mathcal{H}\left(x^{*}, t\right)=0$. Rewrite $\mathcal{H}(x, t)$ as

$$
\mathcal{H}(x, t)=(D+\Delta D(t)) x-(A+\Delta A(t)) \widetilde{g}(x)+\mathcal{H}(0, t),
$$

where $\widetilde{g}(x)=g(x)-g(0)$. By $g_{i} \in \mathcal{I} \mathcal{L}(\alpha)$, it follows that $\widetilde{g}_{i} \in \mathcal{I} \mathcal{L}(\alpha), \widetilde{g}_{i}(0)=0$ and $x_{i} \widetilde{g}_{i}\left(x_{i}\right)>0$ $\left(x_{i} \neq 0\right)$. Set $\Omega_{\mathcal{R}}=\left\{x \in R^{n}:\|x\|<\mathcal{R}\right\}, \mathcal{R}>0$. Define the mapping $\mathcal{H}:[0,1] \times \bar{\Omega} \rightarrow R^{n}$ as

$$
\mathcal{H}(\lambda, x)=(D+\Delta D(t)) x-\lambda(A+\Delta A(t)) \widetilde{g}(x)+\lambda \mathcal{H}(0, t) \quad \forall t,
$$

where $\bar{\Omega}_{\mathcal{R}}=\left\{x \in R^{n}:\|x\| \leq \mathcal{R}\right\}$. By means of Lemma 2.5,

$$
\begin{aligned}
(\widetilde{g}(x))^{T} P \mathcal{H}(\lambda, x)= & (\widetilde{g}(x))^{T} P((D+\Delta D(t)) x+\lambda \mathcal{H}(0, t))-\lambda(\widetilde{g}(x))^{T} P(A+\Delta A(t))(\widetilde{g}(x))= \\
= & (\widetilde{g}(x))^{T} P((D+\Delta D(t)) x+\lambda \mathcal{H}(0, t))- \\
& -\frac{1}{2} \lambda(\widetilde{g}(x))^{T}\left(P(A+H F(t) E)+(A+H F(t) E)^{T} P\right)(\widetilde{g}(x)) \geq \\
\geq & (\widetilde{g}(x))^{T} P((D+\Delta D(t)) x+\lambda \mathcal{H}(0, t))- \\
& -\frac{1}{2} \lambda(\widetilde{g}(x))^{T}\left(P A+A^{T} P+\varepsilon\left(P H(P H)^{T}+\varepsilon^{-1} E^{T} E\right)(\widetilde{g}(x)) .\right.
\end{aligned}
$$

Let $\widetilde{d}_{i}=\min _{t>0}\left(d_{i}-\left|\triangle d_{i}(t)\right|\right)$. By Lemma 2.4, (4) is equivalent to $P A+A^{T} P+\varepsilon(P H)(P H)^{T}+$ $+\varepsilon^{-1} E^{T} E<0$. Hence,

$$
\begin{aligned}
(\widetilde{g}(x))^{T} P \mathcal{H}(\lambda, x) & \geq(\widetilde{g}(x))^{T} P((D+\Delta D(t)) x+\lambda \mathcal{H}(0, t)) \geq \\
& \geq \sum_{i=1}^{n}\left[p_{i}\left(d_{i}+\Delta d_{i}(t)\right)\left|\widetilde{g}_{i}\left(x_{i}\right)\right|\left|x_{i}\right|-\lambda p_{i} \mid \widetilde{g}_{i}\left(x_{i}|| \mathcal{H}(0, t)_{i} \mid\right] \geq\right. \\
& \geq \sum_{i=1}^{n} \widetilde{d}_{i} p_{i}\left|\widetilde{g}_{i}\left(x_{i}\right)\right|\left[\left|x_{i}\right|-\frac{\max _{t>0}\left|\mathcal{H}(0, t)_{i}\right|}{\widetilde{d}_{i}}\right],
\end{aligned}
$$

where $\mathcal{H}(0, t)_{i}$ denotes the $i$ th element of $\mathcal{H}(0, t)$.

By Lemma 2.2, there exist constants $q_{0_{i}}>0$ and $r_{0_{i}}>0$ such that

$$
\left|\widetilde{g}_{i}\left(x_{i}\right)\right| \geq q_{0_{i}} r_{0_{i}}^{\alpha} \quad \forall\left|x_{i}\right| \geq r_{0_{i}}, \quad i=1,2, \ldots, n .
$$

Let $r_{0}=\max _{1 \leq i \leq n} r_{0_{i}}, a=\max _{1 \leq i \leq n} \frac{\max _{t>0}\left|\mathcal{H}(0, t)_{i}\right|}{\widetilde{d}_{i}}, \mathcal{N}_{k}=\left\{n_{1}, \ldots, n_{k}\right\} \subset\{1,2, \ldots, n\}$, $\Omega_{\mathcal{N}_{k}}=\left\{x:\left|x_{i}\right| \leq a, i \in \mathcal{N}_{k}, x \in R^{k}\right\} \forall k<n$. Define

$$
\widetilde{g}_{\mathcal{N}_{k}}(x)=\sum_{i \in \mathcal{N}_{k}} \widetilde{d}_{i} p_{i}\left|\widetilde{g}_{i}\left(x_{i}\right)\right|\left[\left|x_{i}\right|-a\right] .
$$


Noting that $\Omega_{\mathcal{N}_{k}}$ is a compact subset of $R^{k}$ and $\widetilde{g}_{\mathcal{N}_{k}}$ is continuous on $\Omega_{\mathcal{N}_{k}}, \widetilde{g}_{\mathcal{N}_{k}}$ can reach its minimum $\min _{x \in \Omega_{\mathcal{N}_{k}}} \widetilde{g}_{\mathcal{N}_{k}}(x)$ on $\Omega_{\mathcal{N}_{k}}$.

Let $l=\min _{1 \leq i \leq n}\left\{\widetilde{d}_{i} p_{i} q_{0_{i}} r_{0_{i}}^{\alpha}\right\}, \mathcal{M}_{\mathcal{N}_{k}}=\min _{x \in \Omega_{\mathcal{N}_{k}}} \widetilde{g}_{\mathcal{N}_{k}}(x)$ and $\mathcal{M}=\min \left\{\mathcal{M}_{\mathcal{N}_{k}}: \mathcal{N}_{k} \subset\right.$ $\subset\{1,2, \ldots, n\}\}$. Set $\mathcal{R}>\max \left\{\sqrt{n}\left(a-\frac{\mathcal{M}}{l}\right), \sqrt{n} r_{0}\right\}$ and $x \in \partial \Omega_{R}$, then there exist two index sets $\mathcal{N}$ and $\overline{\mathcal{N}}$ such that

$$
\left|x_{i}\right| \leq a, \quad i \in \mathcal{N}, \quad \text { and } \quad\left|x_{i}\right|>a, \quad i \in \overline{\mathcal{N}}
$$

where $\mathcal{N} \cup \overline{\mathcal{N}}=\{1,2, \ldots, n\}$. Furthermore, there exists an index $i_{0}$ in $\overline{\mathcal{N}}$ such that

$$
\left|x_{i_{0}}\right| \geq \frac{\mathcal{R}}{\sqrt{n}} \geq \max \left\{a, r_{0}\right\}
$$

By using (5) and (6), for any $x \in \partial \Omega_{R}$ and $\lambda \in[0,1]$,

$$
\begin{aligned}
(\widetilde{g}(x))^{T} P \mathcal{H}(\lambda, x) & \geq \sum_{i=1}^{n} \widetilde{d}_{i} p_{i}\left|\widetilde{g}_{i}\left(x_{i}\right)\right|\left[\left|x_{i}\right|-\frac{\max _{t>0}\left|\mathcal{H}(0, t)_{i}\right|}{\widetilde{d}_{i}}\right] \geq \\
& \geq \sum_{i \in \overline{\mathcal{N}}} \widetilde{d}_{i} p_{i}\left|\widetilde{g}_{i}\left(x_{i}\right)\right|\left[\left|x_{i}\right|-a\right]+\sum_{i \in \mathcal{N}} \widetilde{d}_{i} p_{i}\left|\widetilde{g}_{i}\left(x_{i}\right)\right|\left[\left|x_{i}\right|-a\right] \geq \\
& \geq \widetilde{d}_{i} p_{i} q_{0_{i_{0}}} r_{0_{i_{0}}}^{\alpha}\left[\left|x_{i_{0}}\right|-a\right]+\mathcal{M} \geq \\
& \geq \widetilde{d}_{i_{0}} p_{i_{0}} q_{0_{i_{0}}} r_{0_{i_{0}}}^{\alpha}\left[\left|x_{i_{0}}\right|-a+\frac{\mathcal{M}}{l}\right] \geq \\
& \geq \widetilde{d}_{i_{0}} p_{i_{0}} q_{0_{i_{0}}} r_{0_{i_{0}}}^{\alpha}\left[\frac{\mathcal{R}}{\sqrt{n}}-a+\frac{\mathcal{M}}{l}\right]>0 .
\end{aligned}
$$

Hence $\mathcal{H}(\lambda, x) \neq 0, x \in \partial \Omega_{R}$ and $\lambda \in[0,1]$. By Lemma 2.3(1),

$$
\operatorname{deg}\left(\mathcal{H}(0, x), \Omega_{\mathcal{R}}, 0\right)=\operatorname{deg}\left(\mathcal{H}(1, x), \Omega_{\mathcal{R}}, 0\right)
$$

i.e., $\operatorname{deg}\left(\mathcal{H}(x, t), \Omega_{\mathcal{R}}, 0\right)=\operatorname{deg}\left((D+\Delta D(t)) x, \Omega_{\mathcal{R}}, 0\right)=\operatorname{deg}|D+\Delta D(t)| \neq 0$, where $\mid D+$ $+\Delta D(t) \mid$ is the determinant of $D+\Delta D(t)$. By Lemma 2.3(2), $\mathcal{H}(x, t)=0$ has at least a solution in $\Omega_{\mathcal{R}}$. Thus, the system (1) has at least an equilibrium point.

Step 2: In this step, the uniqueness of equilibrium point of the system (1) will be proved by the method of contradiction.

Assume that $x_{1}^{*}$ and $x_{2}^{*}$ are two different equilibrium points of the system (1). Then

$$
(D+\Delta D(t))\left(x_{1}^{*}-x_{2}^{*}\right)=(A+\Delta A(t))\left(g\left(x_{1}^{*}\right)-g\left(x_{2}^{*}\right)\right) \text {. }
$$


Hence,

$$
\begin{aligned}
0< & \widetilde{d}_{i} p_{i}\left(g\left(x_{1}^{*}\right)-g\left(x_{2}^{*}\right)\right)^{T}\left(x_{1}^{*}-x_{2}^{*}\right)<\left(g\left(x_{1}^{*}\right)-g\left(x_{2}^{*}\right)\right)^{T} P(D+\Delta D(t))\left(x_{1}^{*}-x_{2}^{*}\right)= \\
& =\left(g\left(x_{1}^{*}\right)-g\left(x_{2}^{*}\right)\right)^{T} P(A+\Delta A(t))\left(g\left(x_{1}^{*}\right)-g\left(x_{2}^{*}\right)\right) \leq \\
& \leq \frac{1}{2}\left(g\left(x_{1}^{*}\right)-g\left(x_{2}^{*}\right)\right)^{T}\left[P(A+H F(t) E)+(A+H F(t) E)^{T} P\right]\left(g\left(x_{1}^{*}\right)-g\left(x_{2}^{*}\right)\right) \leq \\
& \leq \frac{1}{2}\left(g\left(x_{1}^{*}\right)-g\left(x_{2}^{*}\right)\right)^{T}\left(P A+A^{T} P+\varepsilon P H H^{T} P+\varepsilon^{-1} E^{T} E\right)\left(g\left(x_{1}^{*}\right)-g\left(x_{2}^{*}\right)\right)<0 .
\end{aligned}
$$

This is a contradiction. Hence $x_{1}^{*}=x_{2}^{*}$. This implies that the equilibrium point of the system (1) is unique.

Step 3: In this step, by applying Lyapunov function method, the global robust exponential stability of the system (2) will be presented.

Let $\mathcal{F}(x, t)=-(D+\Delta D(t)) x+(A+\Delta A(t)) g(x)+I, \mathcal{F}: R^{n} \rightarrow R^{n}$ is continuous and local bounded on $x$. Hence the existence of the local solution of the system (1) with initial value $x(0)=x_{0}$ on $\left[0, t^{*}\left(x_{0}\right)\right)$ is obvious, where $\left[0, t^{*}\left(x_{0}\right)\right)$ is the maximal right-hand side existence interval of the local solution. This local solution is denoted by $x\left(t, 0, x_{0}\right)$. Let $x^{*}$ be the unique equilibrium point of the system (1). Make a transformation $y(t)=x(t)-x^{*}$, the system (1) is transformed into

$$
\frac{d x}{d t}=-(D+\Delta D(t)) y(t)+(A+\Delta A(t)) \widehat{g}(y(t))
$$

where $y(t)=\left(y_{1}(t), \ldots, y_{n}(t)\right)^{T}, \widehat{g}(y)=\left(\widehat{g}_{1}\left(y_{1}\right), \ldots, \widehat{g}_{n}\left(y_{n}\right)\right)^{T}$, and $\widehat{g}_{i}\left(y_{i}\right)=g_{i}\left(y_{i}+x_{i}^{*}\right)-$ $-g_{i}\left(x_{i}^{*}\right), i=1,2, \ldots, n . y\left(t, 0, y_{0}\right)=x\left(t, 0, x_{0}\right)-x^{*}$ is a solution of the system (7) with with initial values $y(0)=x_{0}-x^{*}$ on $\left[0, t^{*}\left(x_{0}\right)\right)$.

Consider the following Lyapunov function:

$$
V(t)=2 e^{\eta t} \sum_{i=1}^{n} p_{i} \int_{0}^{y_{i}\left(t, 0, y_{0_{i}}\right)} \widehat{g}_{i}(\theta) d \theta,
$$

where $0<\eta<\min _{1 \leq i \leq n} \widetilde{d}_{i}$ is a scalar. Calculating the derivative of $V(t)$ along the solution $y\left(t, 0, y_{0}\right)$ of the system $(\overline{7})$ on $\left[0, t^{*}\left(x_{0}\right)\right)$, it follows that

$$
\begin{aligned}
\frac{d V}{d t}= & 2 \eta e^{\eta t} \sum_{i=1}^{n} p_{i} \int_{0}^{y_{i}\left(t, 0, y_{0_{i}}\right)} \widehat{g}_{i}(\theta) d \theta+ \\
& +2 e^{\eta t}\left(\widehat{g}\left(y\left(t, 0, y_{0}\right)\right)\right)^{T} P\left[-(D+\Delta D(t)) y\left(t, 0, y_{0}\right)+(A+\Delta A(t)) \widehat{g}\left(y\left(t, 0, y_{0}\right)\right)\right] \leq \\
\leq & 2 e^{\eta t}\left(\widehat{g}\left(y\left(t, 0, y_{0}\right)\right)\right)^{T}(\eta P-P(D+\Delta D(t))) y\left(t, 0, y_{0}\right)+ \\
& +e^{\eta t}\left(\widehat{g}\left(y\left(t, 0, y_{0}\right)\right)\right)^{T}\left[P(A+H F(t) E)+(A+H F(t) E)^{T} P\right] \widehat{g}\left(y\left(t, 0, y_{0}\right)\right) \leq \\
\leq & 2\left(\eta-\widetilde{d}_{i}\right) e^{\eta t}\left(\widehat{g}\left(y\left(t, 0, y_{0}\right)\right)\right)^{T} P y\left(t, 0, y_{0}\right)+ \\
& +e^{\eta t}\left(\widehat{g}\left(y\left(t, 0, y_{0}\right)\right)\right)^{T}\left[P A+A^{T} P+\varepsilon P H H^{T} P+\varepsilon^{-1} E^{T} E\right] \widehat{g}\left(y\left(t, 0, y_{0}\right)\right) \leq 0 .
\end{aligned}
$$


This implies $V(t) \leq V(0)$. Hence

$$
2 \sum_{i=1}^{n} p_{i} \int_{0}^{y_{i}\left(t, 0, y_{0_{i}}\right)} \widehat{g_{i}}(\theta) d \theta \leq V(0) e^{-\eta t} \leq V(0)
$$

By (8) and Lemma 2.1, it is easy to derive that $y_{i}\left(t, 0, y_{0}\right), i=1,2, \ldots, n$, are bounded on $\left[0, t^{*}\left(x_{0}\right)\right)$, ie., $y\left(t, 0, y_{0}\right)$ are bounded on $\left[0, t^{*}\left(x_{0}\right)\right)$. By the continuous theorem [26], the system (7) has a solution $y\left(t, 0, y_{0}\right)$ with the initial values $y(0)=x_{0}-x^{*}$ on $[0,+\infty)$. Moreover, by (8)

$$
\lim _{t \rightarrow+\infty} y_{i}\left(t, 0, y_{0_{i}}\right)=0, \quad i=1,2, \ldots, n .
$$

Hence, there exists a constant $T>0$ such that $y_{i}(t) \in\left[-r_{0}, r_{0}\right], i=1,2, \ldots, n, \forall t \geq T$. By Lemma 2.2, when $t \geq T$,

$$
\sum_{i=1}^{n} p_{i} \int_{0}^{y_{i}\left(t, 0, y_{0_{i}}\right)} \widehat{g_{i}}(\theta) d \theta \geq \sum_{i=1}^{n} p_{i} \int_{0}^{\left|y_{i}\left(t, 0, y_{0_{i}}\right)\right|} q_{0}|\theta|^{\alpha} d \theta \geq \frac{p q_{0}}{\alpha+1}\left\{\max _{1 \leq i \leq n}\left|y_{i}\left(t, 0, y_{0_{i}}\right)\right|\right\}^{\alpha+1},
$$

i.e.,

$$
\begin{gathered}
\max _{1 \leq i \leq n}\left|y_{i}\left(t, 0, y_{0_{i}}\right)\right| \leq\left[\frac{\alpha+1}{2 p q_{0}} V(0)\right]^{\frac{1}{1+\alpha}} e^{-\frac{\eta}{\alpha+1} t}, \\
\left\|x\left(t, 0, x_{0}\right)-x^{*}\right\| \leq \sqrt{n}\left[\frac{\alpha+1}{2 p q_{0}} V(0)\right]^{\frac{1}{1+\alpha}} e^{-\frac{\eta}{\alpha+1} t},
\end{gathered}
$$

where $p=\min _{1 \leq i \leq n} p_{i}$. This shows that the equilibrium point of the system (1) is globally exponentially stable, i.e., the system (2) is globally robustly exponentially stable. The proof is completed.

Remark 2. (i) The condition (4) in Theorem 3.1 is a LMI if $\varepsilon$ is given. Hence when $\varepsilon$ is given, the problem to seek a feasible diagonal matrix $P$ which is used to check the global exponential robust stability of the system (2) can be solved by using appropriate LMI solver in the Matlab [25].

(ii) Let $\mathbb{P}=\left\{\left(\varepsilon, p_{1}, p_{2}, \ldots, p_{n}\right) \mid \varepsilon, p_{i}>0, i=1,2, \ldots, n, \mathbb{E}<0\right\}$. Generally, it is difficult to obtain the detail of $\mathbb{P}$. However, the bounds of $\mathbb{P}$ can be determined if the following optimization problems can be solved

$$
\begin{aligned}
& \text { Maximize } \varepsilon, p_{1}, p_{2}, \ldots, p_{n} \\
& \text { subject to }\left(\varepsilon, p_{1}, p_{2}, \ldots, p_{n}\right) \in \mathbb{P} \text {, } \\
& \text { Minimize } \varepsilon, p_{1}, p_{2}, \ldots, p_{n} \\
& \text { subject to }\left(\varepsilon, p_{1}, p_{2}, \ldots, p_{n}\right) \in \mathbb{P} \text {. }
\end{aligned}
$$

(9) and (10) are quasiconvex optimization problems and can be easily solved by using the Matlab Toolbox. Let $\bar{\varepsilon}, \overline{p_{1}}, \overline{p_{2}}, \ldots, \overline{p_{n}}$ and $\underline{\varepsilon}, \underline{p_{1}}, \underline{p_{2}}, \ldots, \underline{p_{n}}$ be the feasible solutions of (9) and (10) 
respectively, $\mathbb{D}=\left\{\left(\varepsilon, p_{1}, p_{2}, \ldots, p_{n}\right) \mid \underline{\varepsilon}<\varepsilon<\bar{\varepsilon}, p_{i}<p_{i}<\overline{p_{i}}, i=1,2, \ldots, n\right\} . \mathbb{D}$ is a polytopic domain. Obviously, based on Theorem 3.1, the vector $\left(\varepsilon, p_{1}, p_{2}, \ldots, p_{n}\right)$ which ensues that the system (2) is globally robustly exponentially stable should be contained in $\mathbb{D}$. If $\mathbb{D}=\emptyset$, then the global exponential robust stability of the system (2) can not be checked by using Theorem 3.1.

4. Illustrative examples. Consider the second-order neural network (1) described by $D=$ $=\operatorname{diag}(1,1), \Delta D(t)=\operatorname{diag}(0.5 \sin t, 0.4 \cos t), A=\left(\begin{array}{cc}-1 & -2 \\ 2 & -1\end{array}\right), E=H=\left(\begin{array}{cc}0,5 & 0 \\ 0 & 0,5\end{array}\right)$, $F(t)=\left(\begin{array}{cc}0,5 \sin t & 0 \\ 0 & 0,5 \cos t\end{array}\right), I=(0,0)^{T}$ and $g(\theta)=\theta^{3} \in \mathcal{I} \mathcal{L}(3)$. It is easy to check that $(0,0)^{T}$ is the equilibrium point of the network. Choose $\varepsilon=1$. Solving the LMI in (4) by using appropriate LMI solver in the Matlab, the positive diagonal matrix $P$ could be as

$$
P=\left(\begin{array}{cc}
1,1580 & 0 \\
0 & 1.1580
\end{array}\right)
$$

By Theorem 3.1, the unique equilibrium point of this neural network is global exponential stable.

Figures 1 and 2 display the time-domain behavior of the network. It can be seen that state trajectories of this network with 10 initial values converge to the equilibrium point $(0,0)^{T}$. This is in accordance with the conclusion of Theorem 3.1.

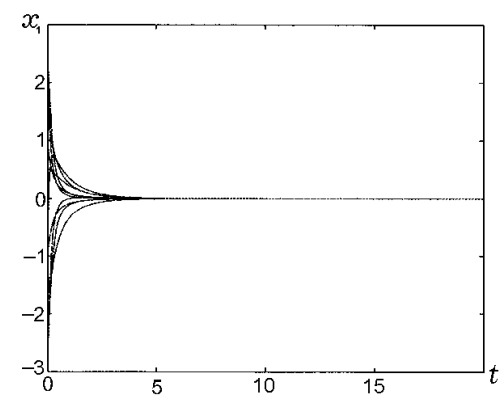

Fig. 1. The state trajectory $x_{1}$ of the network with 10 initial values in Example 1.

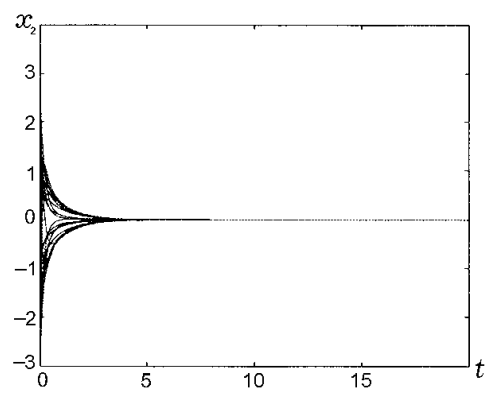

Fig. 2. The state trajectory $x_{2}$ of the network with 10 initial values in Example 1. 
Example 2. Consider the third-order neural network (1) descried by

$$
\begin{gathered}
D=\operatorname{diag}(1,1,1), \quad \Delta D(t)=\operatorname{diag}(0,5 \sin t, 0,4 \cos t, 0,5 \sin t), \\
A=\left(\begin{array}{ccc}
-3 & -2 & 1 \\
2 & -1 & 1 \\
-1 & 2 & -3
\end{array}\right), \quad H=E=\left(\begin{array}{ccc}
0,5 & 0 & 0 \\
0 & 0.5 & 0 \\
0 & 0 & 0.5
\end{array}\right), \\
F(t)=\left(\begin{array}{ccc}
0,2 \sin t & 0 & 0 \\
0 & 0,5 \cos t & 0 \\
0 & 0 & 0,4 \sin t
\end{array}\right), \quad I=(0,0,0)^{T} \text { and } g(\theta)=\arctan (\theta) \in \mathcal{I} \mathcal{L}(1) .
\end{gathered}
$$

It is easy to check $(0,0,0)^{T}$ is the equilibrium point of the network. Choose $\varepsilon=1$. Solving the LMI in (4) by using appropriate LMI solver in the Matlab, the positive diagonal matrix $P$ could be as

$$
P=\left(\begin{array}{ccc}
1,7917 & 0 & 0 \\
0 & 1,3466 & 0 \\
0 & 0 & 0,7271
\end{array}\right)
$$

By Theorem 3.1, the unique equilibrium point of this neural network is global exponential stable.

Figures 3, 4 and 5 display the time-domain behavior of the network. It can be seen that state trajectories of this network with 10 initial values converge to the equilibrium point $(0,0,0)^{T}$. This is in accordance with the conclusion of Theorem 3.1.

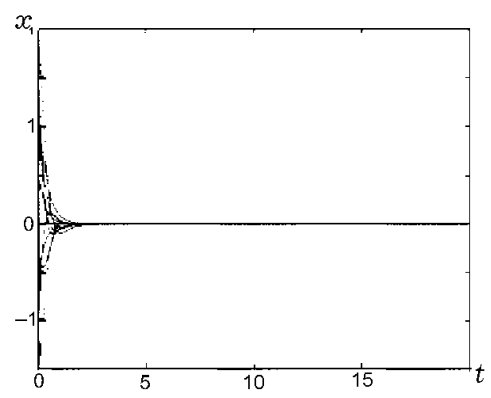

Fig. 3. The state trajectory $x_{1}$ of the network with 10 initial values in Example 2.

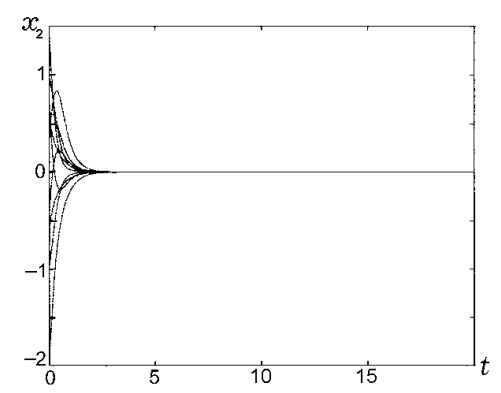

Fig. 4. The state trajectory $x_{2}$ of the network with 10 initial values in Example 2. 


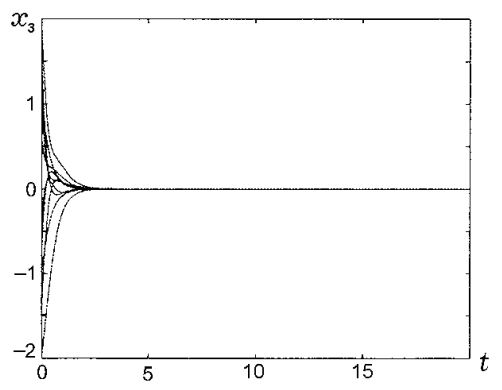

Fig. 5. The state trajectory $x_{3}$ of the network with 10 initial values in Example 2.

5. Conclusion. In this paper, a new class of Hopfield neural networks with norm-bounded parameter uncertainties and inverse Hölder neuron activation functions has been presented. A sufficient condition to the existence, uniqueness and global robust stability of equilibrium point for such neural networks has been derived by employing Brouwer degree properties and Lyapunov method. The results obtained in this paper are given in the form of LMIs, which can be checked and applied easily in practice. Two numerical examples have been also exploited to show the use of the proposed LMI-based stability criteria.

As is well known, when neuron activation functions are non-Lipschitz functions, it is possible that the neural network system has not the global solution and / or the equilibrium point. Hence, this leads to difficulty in solving the problem of the stability for neural networks with non-Lipschitz activation functions. In the future, when the neuron activations are other nonLipschitz activation functions, the stability, particularly robust stability for neural networks with the norm-bounded parameter uncertainties or the interval uncertainty will be an important yet challenging problem. It will be expected to be solved by the researchers in this filed.

1. Singh V. A new criterion for global robust stability of interval delayed neural networks // J. Comput. Appl. Math. - 2008. - 221. - P. 219-225.

2. Singh $V$. Improved global robust stability for interval-delayed hopfield neural networks // Neural. Process Lett. - 2008. - 27. - P. 257-265.

3. Zhang B., Xu S., Li Y. Delay-dependent robust exponential stability for uncertain recurrent neural networks with time-varying delays // Int. J. Neural. Syst. - 2007. - 17. - P. 207 - 218.

4. Zhang H., Wang Z., Liu D. Robust stability analysis for interval Cohen-Grossberg neural networks with unknown time-varying delays // IEEE Trans Neural Network. - 2008. - 19. - P. 1942-1955.

5. Zhao W., Zhu $Q$. New results of global robust exponential stability of neural networks with delays // Nonlinear Anal.: Real World Appl. - 2010. - 11. - P. 1190-1197.

6. Kwon O., Park J., Lee S. On roust stability for uncertain neural networks with interval time-varying delays // IET Control Theory Appl. - 2008. - 2. - P. 625-634.

7. Gau R., Lien C., Hsieh J. Global exponential stability for uncertain cellular neural networks with multiple time-varying delays via LMI approach // Chaos Soliton Fract. - 2007. - 32. - P. 1258-1267.

8. Shao J., Huang T., Zhou S. An analysis on global robust exponential stability of neural networks with timevarying delays // Neurocomputing. - 2009. - 72. - P. 1993 -1998.

9. Zheng C., Zhang H., Wang Z. Novel delay-dependent criteria for global robust exponential stability of delayed cellular neural networks with norm-bounded uncertainties // Neurocomputing. -2009 . - 721. P. $744-1754$. 
10. Qiu J., Zhang J., Wang J., Xia Y., Shi P. A new global robust stability criteria for uncertain neural networks with fast time-varying delays // Chaos Soliton Fract. - 2008. - 37. - P. 360-368.

11. Wang Z., Zhang $H$., $Y u W$. Robust exponential stability analysis of neural networks with multiple time delays // Neurocomputing. - 2007. - 70. - P. 2534-2543.

12. Senan S., Arik S. New results for global robust stability of bidirectional associative memory neural networks with multiple time delays // Chaos, Solitons and Fractals. - 2009. - 41. - P. 2106-2114.

13. Sheng L., Yang H. Robust stability of uncertain Markovian jumping Cohen-Grossberg neural networks with mixed time-varying delays // Chaos, Solitons and Fractals. - 2009. - 42. - P. 2120-2128.

14. Wang $Z$., Zhang $H$., $Y u$ W. Robust stability of Cohen-Grossberg neural networks via state transmission matrix // IEEE Trans Neural Network. - 2009. - 20. - P. 169-174.

15. Su W., Chen $Y$. Global robust exponential stability analysis for stochastic interval neural networks with timevarying delays // Commun. Nonlinear Sci. Numer. Simulat. - 2009. - 14. - P. 2293-2300.

16. Wang L., Zhang Y., Zhang Z., Wang Y. LMI-based approach for global exponential robust stability for reaction-diffusion uncertain neural networks with time-varying delay // Chaos, Solitons and Fractals. -2009. - 41. - P. $900-905$.

17. Zhang R., Wang L. Global exponential robust stability of interval cellular neural networks with S-type distributed delays // Math. Comput. Model. - 2009. - 50. - P. 380-385.

18. Zhang J., Peng S., Qiu J. Robust stability criteria for uncertain neutral system with time delay and nonlinear uncertainties // Chaos, Solitons and Fractals. - 2008. - 38, № 1. - P. 160 - 167.

19. Chen Y., Xue A., Lu R., Zhou S. On robustly exponential stability of uncertain neutral systems with timevarying delays and nonlinear perturbations // Nonlinear Anal. - 2008. - 68. - P. 2464-2470.

20. Zheng C., Jing X., Wang Z., Feng J. Further results for robust stability of cellular neural networks with linear fractional uncertainty // Commun Nonlinear Sci. Numer. Simulat. - 2009. / doi:10.1016/j.cnsns.2009.11.007.

21. Wu H., Tao F., Qin L., Shi R., He L. Robust exponential stability for interval neural networks with delays and non-Lipschitz activation functions // Nonlinear Dynam. - 2010 // DOI 10.1007 / s11071-010-9926-9.

22. $W u H .$, Xиe $X$. Stability analysis for neural networks with inverse Lipschitzan neuron activations and impulses // Appl. Math. Model. - 2008. - 32. - P. 2347-2359.

23. activations, Nonlinear Anal.: Real World Appl. - 2009. - 10. - P. 2297-2306.

24. Deimling K. Nonlinear functional analysis. - Berlin: Springer, 1985.

25. Boyd S., Ghaoui L. E., Feron E., Balakrishnan V. Linear matrix in equalities in system and control theory. Philadelphia, PA: SIAM, 1994.

26. Miller P., Michel A. Differential equations. - New York: Academic, 1982.

Received 06.01.11, after revision - 25.04 .11 